\title{
A CONSENSUS STATEMENT - PART I. RECOMMENDATIONS FOR THE MANAGEMENT OF CHRONIC VENOUS DISEASE (CVD) IN INDIA AND THE KEY ROLE OF PRIMARY CARE DOCTORS
}

\author{
Devendra Dekiwadia', Ravul Jindal2, Roy Varghese ${ }^{3}$, Harinder Singh Bedi4, \\ Shoaib Padaria ${ }^{5}$, Malay Patel ${ }^{6}$ \\ 'Dekiwadia Hospital, Rajkot, India \\ 2Fortis Hospital, Mohali, India \\ 3 Jubilee Hospital, Kerala, India \\ ${ }^{4}$ CMC Ludhiana, Ludhiana, India \\ 5 Jaslok Hospital, Mumbai, India \\ ${ }^{6}$ First Choice Vascular Clinic, Gujarat, India
}

\section{REVIEW PAPER}

Phlebological Review 2016; 24, 1: 7-19

DOI: 10.5114/pr.2016.61533

Submitted: 24.05 .2016

Accepted: 26.05 .2016

\begin{abstract}
This consensus intentionally focuses only on the acquired chronic venous disease (CVD). One of the biggest risk factors for CVD is prolonged standing. Certain occupations that require people to stand for a long time (like nursing, teaching, traffic police work, office work, housework, etc.) increase the risk of acquired CVD.

In the female gender there is an increased risk of post-partum CVD.

Based on this we will conduct a nationwide survey of the disease prevalence, pattern, and epidemiology to give a guideline useful for the Indian set up, as well as many countries other than India having diversified conditions.

In India, awareness of the warning symptoms of CVD among the general population is far from satisfactory.

This lack of awareness leads to a delay in receiving medical advice from primary care doctors and a delay in adequate treatment.

A high prevalence of CVD has been documented in India and hence studies need to be conducted in different parts of India to develop an understanding at a national level of the pattern of chronic venous disease and our Indian observation.

This will help us search in our country for a better way to understand and treat the disease.

This is because India is a multi-ethnic, multi-cultural country with vast geographic variance, which could influence the patients' lifestyle and its impact on CVD. There is now an urgent need to undertake health education measures about the awareness of risk factors and early warning signs of CVD in the community, so that people, and affected subjects specifically, can receive early therapy through modern means.
\end{abstract}

Key words: medical treatment, recommendation, India, MPFF, chronic venous disease.

\author{
ADDRESS FOR CORRESPONDENCE \\ Devendra Dekiwadia \\ Dekiwadia Hospital \\ Canal Road \\ 360001 Rajkot, India \\ e-mail: dekiwadia@rediffmail.com
}

\section{WHAT DOES THE TERM “CHRONIC VENOUS DISEASE" COVER?}

The term "chronic venous disease" (CVD) covers a full spectrum of venous conditions of lower limbs, ranging from patients with early symptoms, like telangiectasia (reticular veins), leg pain, or oedema of the foot, to complications like venous leg ulcers. In the early stages usually only symptoms appear, then as the disease progresses it is accompanied by signs.

Venous symptoms are defined as tingling, aching, burning sensation, muscle cramps, swelling, sensations of throbbing or heaviness, itching skin, restless legs, leg tiredness, or fatigue. These may be exacerbated during the course of the day by prolonged standing or by heat, and relieved with leg rest, elevation, or both. Venous signs are visible manifestations of chronic venous disease, which include [1]:

- Oedema of the dorsum of foot, the entire foot, possibly extending to the leg;

- Frank varicose veins: this may or may not be associated with telangiectasia or reticular veins;

- Skin changes: dryness, frank eczema, darkened, thickened, hard, scarred, and ulcers. 


\section{BURDEN OF CHRONIC VENOUS DISEASE}

In the current scenario, the awareness and the willingness to get CVD treated is very low amongst patients in India. Chronic venous disease is also often overlooked by healthcare providers for various reasons. One of the most important factors is a lack of proper guidance in terms of diagnosing the disease and its severity, selecting the best treatment for the patients, and follow-up. The answer lies in understanding the various presenting manifestations of primary and secondary venous disorders.

On a global perspective 1 out of 5 people in the world suffer from CVD. This can be mainly attributed to occupation, lifestyle changes, and some environmental factors. In developed nations, CVD is most often brought to a doctor's attention only when it becomes a cosmetic concern, and in India at a much later stage, i.e. when it affects the quality of life and begins to limit the functioning of the lower limbs. This clearly indicates that patients rarely seek treatment at the early stages of the disease. Hence CVD remains an iceberg phenomenon [2].

The term CVD can be defined as "any morphological and functional abnormalities of the venous system of long duration caused by venous dilation or venous reflux indicating the need for investigation and/or care". Whereas chronic venous insufficiency (CVI) is a term reserved

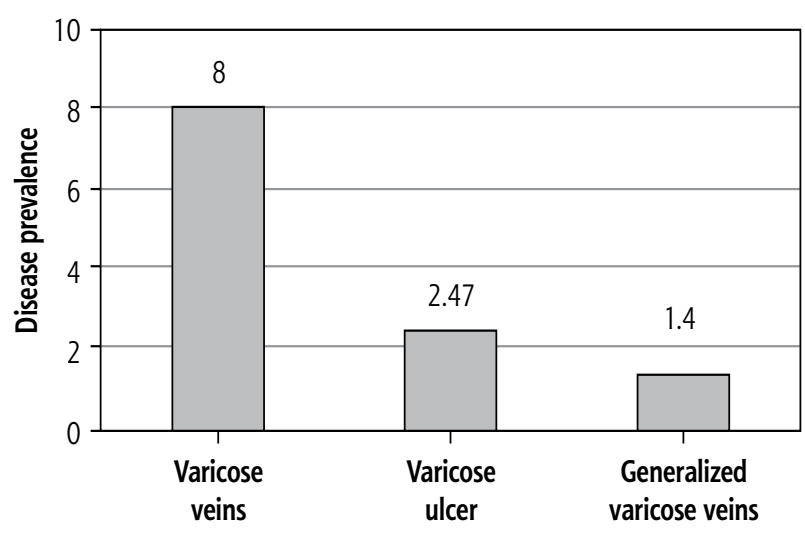

Fig. 1. Indian prevalence of varicose veins and venous ulcers

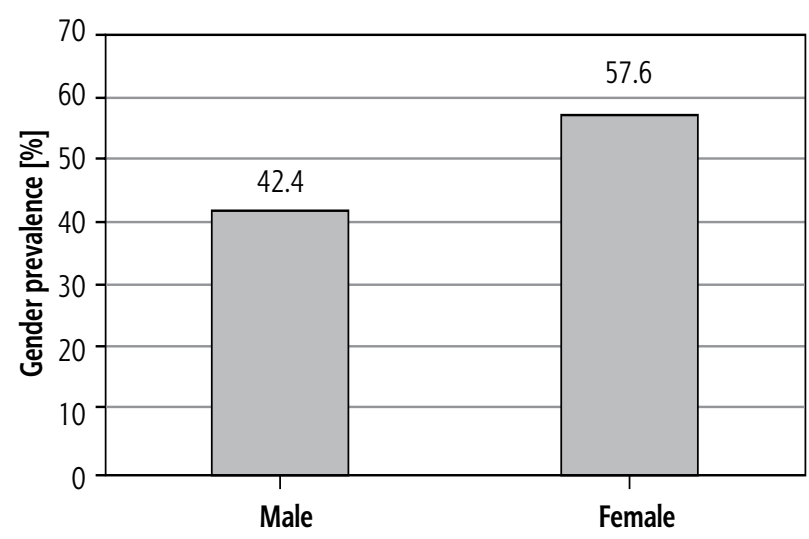

Fig. 2. Estimated gender prevalence of CVD in India for advanced CVD, which is applied to functional abnormalities of the venous system producing oedema, skin changes, or venous ulcers [3].

Chronic venous disease can have an impact on the lives of the people whose occupation involves standing for long hours like the teachers, nursing staff, flight attendants, traffic policemen, bar workers, and many more. The duration of standing has been recognised as a vital contributor to CVI [2]. A study indicated that workers who stood for more than $50 \%$ of working hours (four hours) exhibited a higher frequency of CVI than workers who spent less time standing [4]. Another cross-sectional study was conducted amongst traffic policemen in Ahmedabad city, India, to study the socio-demographic profile of traffic policemen and their health problems. This study concluded that $2.91 \%$ of respondents had varicose veins, while in the study conducted by Satpathy et al., $4.17 \%$ had varicose veins of the legs [5].

\section{The epidemiology: Indian perspective}

The epidemiology of venous disease has received little attention in developing countries [6].

- The prevalence of CVD in the western adult population is estimated to be seen in $25-33 \%$ of women and $10-20 \%$ of men [7].

- The prevalence of chronic leg ulcers increases dramatically with age. Leg ulceration affects about $1 \%$ of the adult population and $3.6 \%$ of people older than 65 years [8].

- In a study conducted by Malhotra et al., $8 \%$ of the South Indians had generalised varicose veins and $2.47 \%$ had complications such as pigmentation, varicose ulcer, oedema, and eczema. Only $1.4 \%$ of the North Indian men had generalised varicose veins (Fig. 1) [9].

- In a prospective observational study of six months (No. of patients $=308$ ) involving 11 major cities of India, it was found that CVD was more prevalent at the age of $42.9 \pm 11.5$ years $[10]$.

- Chronic venous disease tends to affect women more than men. The prevalence of CVD in India is estimated at $42.4 \%$ in males and $57.6 \%$ in females (Fig. 2) [10].

- The most common symptoms at the time of presentation in CVI patients were lower limb pain, swelling, heaviness, and cramps. Observed symptom intensity was $1.5 \pm 0.8$ for swelling, $1.5 \pm 0.8$ for pain, $1.8 \pm 0.7$ for heaviness, and $1.5 \pm 0.9$ for cramps [10].

- Quality of life (QoL) can be considerably compromised in patients who are suffering from chronic venous insufficiency [11]. The high prevalence of CVD, cost of investigation and treatment, and loss of working days implies that CVD has a considerable socioeconomic impact. In western countries the cost due to venous disease represented $2.6 \%$ of the total healthcare budget. Despite this, in India there is little awareness or information on CVI [12]. 


\section{Chronic venous disease: problems peculiar to the Indian scenario}

- Many patients in India consider varicose veins as "nerves" and hence are scared of treatment because of a fear that they may not be able to walk again [12].

- The worst sufferers of venous insufficiency come from the group engaged in occupations that involve long periods of standing, like police force or staff in a grocery shop, bakery, hotels, etc. who do not have the awareness or the means to change their occupation.

- Deep vein thrombosis (DVT) has always been under-recognised, under-diagnosed, and under-treated in India. These patients present very late due to severe stasis changes. Physicians should remember that the post-thrombotic syndrome seen today could be the neglected DVT of yesterday.

- In some cases, even after surgery, varicose veins can recur. Fear of surgery and fear of recurrence are the main reasons why Indian patients typically come very late for treatment.

- Requests for surgery from a cosmetic point of view rare. Patients consult during the advanced stage, which leads to further complications [13].

\section{Primary acquired chronic venous disease}

Chronic venous disease appears to be related to an obesity-promoting Western lifestyle, which has poor intake of dietary fibre and low physical activity. Evidence suggests that avoidance of these risk factors may reduce the incidence of venous disorders [14]:

- A body mass index (BMI) $\geq 30 \mathrm{~kg} / \mathrm{m}^{2}$ in women increases the risk for CVD significantly [14].

- Other authors have found an association between severe obesity $\left(\mathrm{BMI}>40 \mathrm{~kg} / \mathrm{m}^{2}\right)$ and increasing limb symptoms without anatomic evidence of venous disease, suggesting that the obesity itself contributed to venous insufficiency [15].

There are many factors such as genetics, gender, and obesity, which contribute to varicose veins. However, one of the biggest risk factors for varicose veins is prolonged standing or sitting. Certain occupations that require people to sit or stand for a long time can lead to venous disease [16].

- Office workers: Mostly, office workers sit uninterrupted at their desks for much of the day. Since it is an unhealthy way to work, a sedentary desk job can be a contributing cause of varicose veins.

- Nurses: Nursing requires long periods of standing. Whether it is taking vitals, interviewing patients, checking up on patients, or assisting doctors during surgery, nurses spend the majority of the day on their feet. Also, many nurses wear compression stockings to prevent varicose veins.

- Factory workers: People working in a factory or on an assembly line stand on their feet for a long time every day, which can cause varicose veins.

\section{NEED FOR A COMMON LANGUAGE IN UNDERSTANDING AND MANAGING CHRONIC VENOUS DISEASE IN INDIA}

In a consensus, it is important to have a common language while describing the disease. The international ad hoc committee of the American Venous Forum published CEAP classification in 1994 and was endorsed by the Society for Vascular Surgery. It was revised in 2004 and is a widely endorsed classification system for clinical papers reporting on CVD (Table 1) [17].

The CEAP classification includes [1]:

- A Clinical assessment (C);

- An Etiological assessment of the patient's disease (E);

- An Anatomical assessment of the location of the pathology (A);

- And the Pathophysiological basis for the underlying disease $(\mathrm{P})$.

Clinical signs form the basis of the clinical component of CEAP, which is scored from 0 (no evidence of venous disease) to 6 (active ulceration). Although CEAP is generally considered to represent increasing disease severity, this should not be considered as a linear progression or severity score. Unlike the Widmer and Porter classifications, the CEAP classification allows more details to be recorded.

Symptoms of CVD, including aching, pain, tightness, skin irritation, heaviness, and muscle cramps are denoted by the letter $\mathrm{S}$ in subscript, for example $\mathrm{C} 2_{\mathrm{s}}$ (symptomatic) and the letter $\mathrm{A}$, if asymptomatic, for example $\mathrm{C} 2{ }_{\mathrm{A}}$. Even if skin changes have occurred, a patient may be asymptomatic, for example $\mathrm{C}_{\mathrm{A}}$ [18].

The clinical features increase with time and consist of telangiectasia, varicose veins, oedema, hyperpigmentation, eczema, white atrophy, lipodermatosclerosis, changes in the nails, and ulceration (Fig. 3) [19].

Table 1. Clinical classification system for chronic venous disease

\begin{tabular}{cc}
\hline $\begin{array}{c}\text { CEAP clinical } \\
\text { classification } \\
\text { of CVD }\end{array}$ & Clinical classification \\
\hline$C_{0}$ & No visible or palpable signs of venous disease \\
\hline$C_{1}$ & Telangiectasia or reticular veins \\
\hline$C_{2}$ & Varicose veins \\
\hline$C_{3}$ & Oedema \\
\hline$C_{4 a}$ & Pigmentation or eczema \\
\hline$C_{4 b}$ & Lipodermatosclerosis or atrophie blanche \\
\hline$C_{5}$ & Healed venous ulcer \\
\hline$C_{6}$ & Active venous ulcer \\
\hline$S$ & Symptomatic - pain, tightness, skin irritation, heaviness, \\
& and muscle cramps \\
\hline$A$ & Asymptomatic \\
\hline
\end{tabular}


Tools to evaluate the improvement in symptoms of chronic venous disease

\section{Venous clinical severity score (VCSS)}

The VCSS offers a broad quantification of the severity of venous disease. A total of 10 clinical characteristics are evaluated and graded from absent (score 0) to severe (score 3). It was developed to assess the progression of CVD and also to give additional weight to more severe clinical disease (C4-C6) [18].

\section{Venous segmental disease score (VSDS)}

The VSDS takes into account the anatomical and pathophysiological mechanisms involved in the presentation of CVD. VSDS accounts for anatomical location and nature (reflux or obstruction) of venous disease, providing a global assessment of pathophysiological disease severity [18].

\section{The chronic venous insufficiency questionnaire (CIVIQ)}

The chronic venous insufficiency questionnaire (CIVIQ) is a 20-item self-reporting QoL tool covering four dimensions: physical, psychological, social functioning, and pain [11].

\section{RECOMMENDED PROCESS FOR DIAGNOSIS OF CHRONIC VENOUS DISEASE BY A PRIMARY CARE DOCTOR}

\section{History}

- Symptoms of venous insufficiency include aching, pain, tightness, skin irritation, pruritus, heaviness, tingling, muscle cramps, and cosmetically unsatisfying varicose veins. Symptoms might worsen with prolonged standing or at the end of the day.

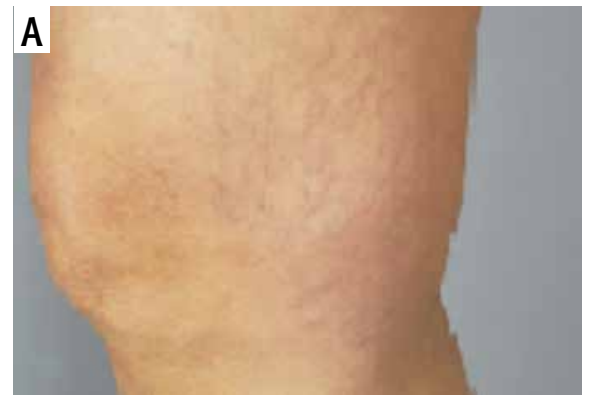

C1: Telangiectasia

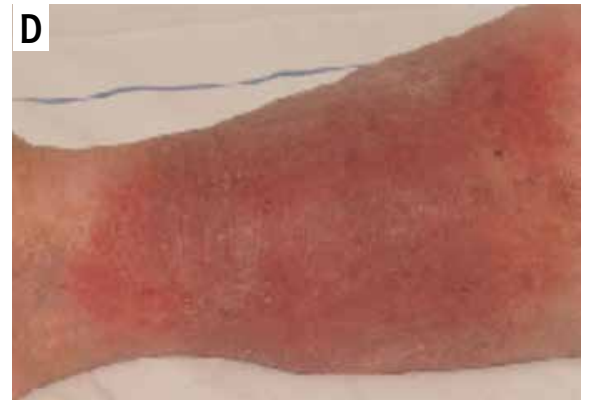

C4a: Eczema

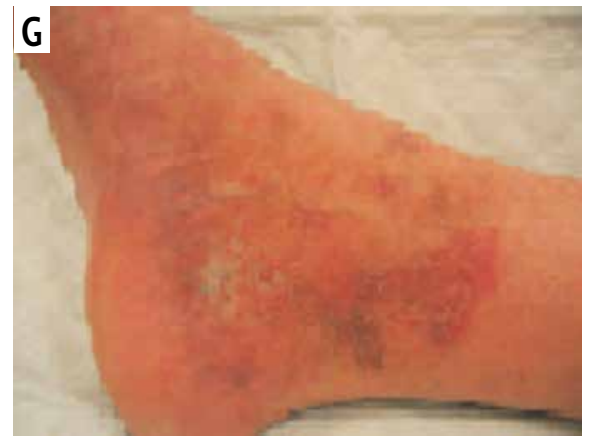

C4b: Atrophie blanche

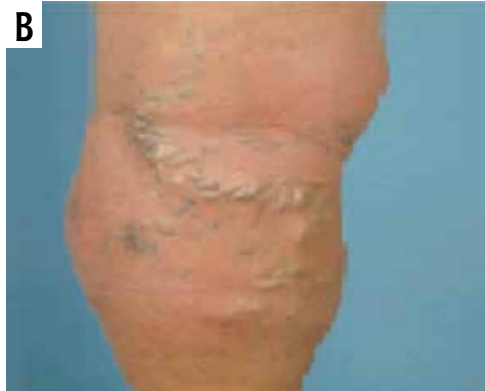

C2: Varicose veins

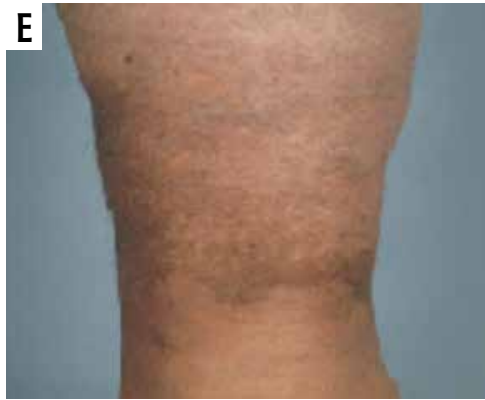

C4a: Pigmentation

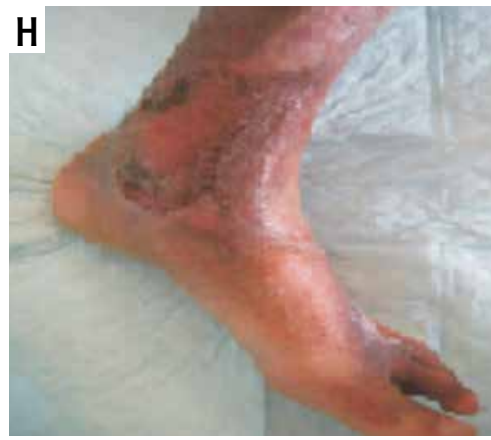

C5: Healed venous ulcer

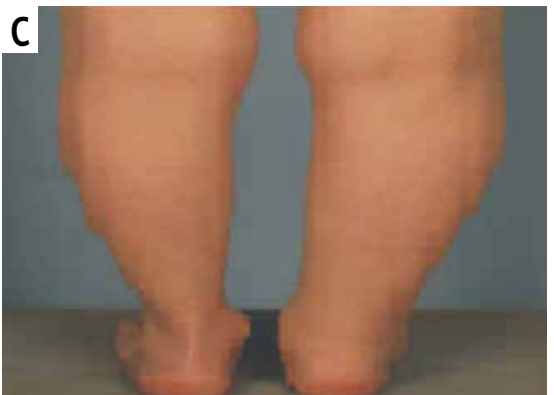

C3: Oedema

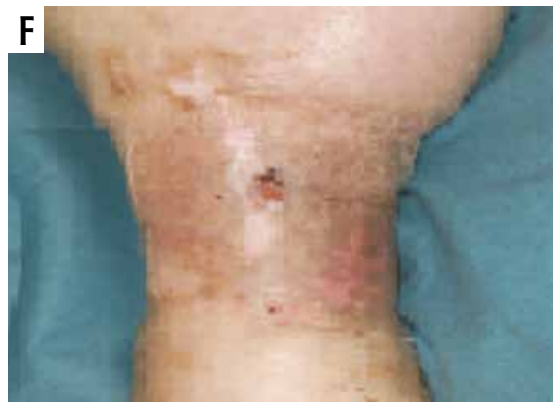

C4b: Lipodermatosclerosis

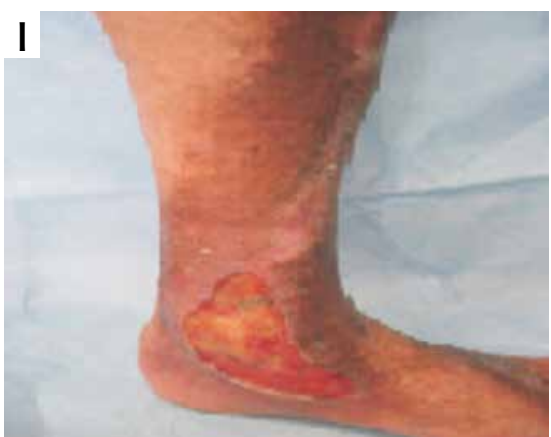

C6: Active venous ulcer (same patient as Fig. 3H)

Fig. 3. Clinical features of chronic venous disease 
- In case of severe venous insufficiency, the patient can present with complaints of oedema, skin changes, or ulceration.

- Specific features of the pain that should be noted include the degree to which the pain interferes with the patient's occupation or lifestyle as well as the amount of time that the patient can stand before the onset of pain or swelling.

- A positive family history of varicose veins is seen in over one third of patients. It is crucial to note whether there has been a personal history of DVT or pulmonary embolism. Some patients may not be able to provide this information directly, and it may be elicited only by asking specific questions regarding a history of leg swelling, previous operations, lower extremity injuries, prolonged bed rest, chest pain, haemoptysis, or anticoagulant use.

- A careful history of past treatment for varicose veins, including operative and percutaneous procedures, should be recorded [20].

\section{Physical examination}

- Examine the patient in the standing position and suitably undressed to complete the examination of the entire lower extremity from the groin to the toes.

- Initial examination begins with a careful inspection and palpation of the legs. The location and distribution of all major subcutaneous varicosities should be noted.

- Varicosities in the main saphenous trunk and spider veins should be noted. Large varicosities over known sites of perforating veins should be identified.

- Palpation of the legs should also be performed to detect temperature differences between the legs, areas of induration, and the presence of firm subcutaneous cords, which may be the squeal of prior episodes of superficial thrombophlebitis [20].

\section{Other investigations}

- Colour Doppler is considered as a gold standard in the evaluation of the venous patient.

- For the patient with deep vein thrombosis, apart from venous Doppler, other investigations will include CBC (to evaluate $\mathrm{Hb}$ possible leukaemia), urea, creatinine, complete urinalysis to evaluate protein urea, and liver function tests.

- These include plethysmography, isotope studies, venography, and intravascular ultrasound, which are not widely available and also may not be very relevant from a clinical studies point of view [13].

\section{TREATMENT OPTIONS PROPOSED BY INDIAN EXPERTS IN CHRONIC VENOUS DISEASE}

A variety of treatment methods are currently available for patients with CVD, based on the severity of the disease and expert guidance. The treatment options are mentioned below.

\section{Lifestyle management}

\section{Exercise programme}

- Abnormalities in the calf and foot muscle pump functions play a significant role in the pathophysiology of CVI.

- Padberg et al. concluded that structured exercise to re-establish calf muscle pump function in CVI may prove beneficial as a supplemental therapy to medical and surgical treatment in advanced disease.

- Graded exercise programs have been used in an effort to rehabilitate the muscle pump and improve the symptoms of CVI [21].

\section{Leg elevation}

- Leg elevation improves venous stasis, provides symptomatic relief, reduces leg oedema, and promotes healing of ulcers in patients with CVD.

- Leg elevation in CVD patients classified as C3-C6 has been shown to reduce leg volume and venous pressure, and to enhance the microcirculatory flow velocity in lipodermatosclerotic skin.

- In the past, elevation has been used as the main measure to heal venous leg ulcers or as an adjunctive measure in patients wearing elastic bandages. It is still practiced in combination with antibiotics and debridement in cases of infected ulcers where compression cannot be tolerated because of pain associated with cellulitis in the surrounding areas [18].

\section{Leg massage}

- This method can be part of a multi-component regimen aimed at reducing tissue oedema by applying deep massage around the ulcer area, before formal compression is applied, or as an adjunct.

- Leg massage entails a form of light massage over elastic compression with a stocking [18].

\section{Pharmacological treatments available in India}

Medical treatment for CVD is a topic of continuing debate. Veno-active drugs are widely prescribed in most countries and are classified as natural and synthetic drugs, such as MPFF and calcium dobesilate, pentoxifylline, HCSE, etc. These drugs decrease capillary permeability, diminish release of inflammatory mediators, or improve venous tone. Pentoxifylline, a non-veno-active drug, reduces white cell activation. Also, acetylsalicylic acid is considered because of its anti-inflammatory effect and platelet inhibiting function. 


\section{Micronised purified flavonoid fraction (MPFF)}

- MPFF is an oral phlebotropic drug consisting of $90 \%$ micronised diosmin and $10 \%$ flavonoids expressed as hesperidin. It improves venous tone and lymphatic drainage, and reduces capillary hyper-permeability by protecting the microcirculation from inflammatory processes.

- MPFF is a well-established and well-tolerated treatment option in patients with CVI, venous ulcers, or acute or chronic internal haemorrhoids.

- MPFF is considered as a first-line treatment of oedema and the symptoms of CVI in patients in any stage of the disease.

- In more advanced disease stages, MPFF may be used in conjunction with sclerotherapy, surgery, and/or compression therapy, or as an alternative treatment when surgery is not indicated or is unfeasible [22].

\section{Mechanism of action}

MPFF is thought to have numerous modes of action including [23]:

- Inhibition of noradrenaline degradation by catechol-O-methyltransferase and thus indirectly increasing venous tone;

- Inhibition of leukocyte adhesion and activation and therefore reducing inflammation;

- Inhibition of platelet function;

- Increased lymphatic drainage.

\section{Results from RELIEF study}

- It was the largest study conducted in Indian patients; a total of 308 patients from 11 cities of India were treated for six months with MPFF at $1000 \mathrm{mg}$ daily. A total of $53.9 \%$ showed regression in the CEAP severity stage; $29.5 \%$ showed no change in CEAP but showed improvement in symptoms; $8.1 \%$ showed worsening of

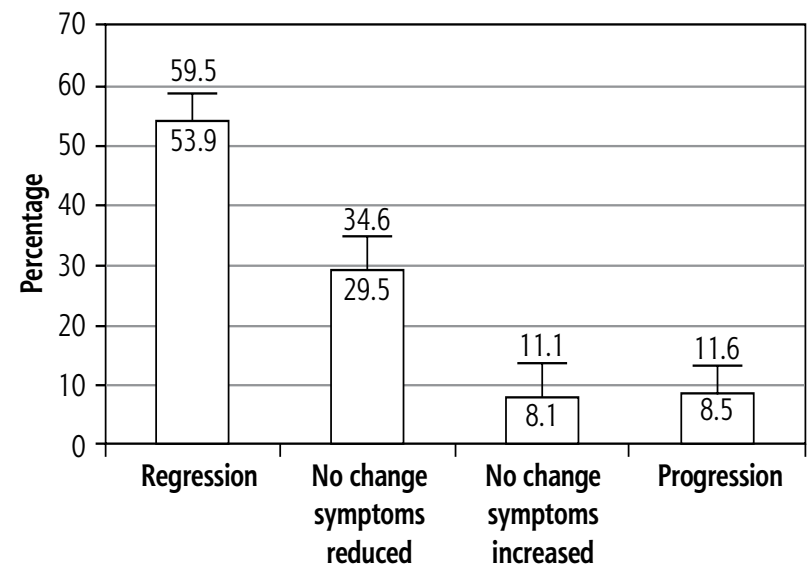

Fig. 4. Patients with chronic venous insufficiency of the leg showing change in CEAP severity stage after six months of treatment with MPFF. $T=95 \%$ confidence interval symptoms while remaining in the same CEAP severity stage; and $8.5 \%$ had progression to the next CEAP severity stage (Fig. 4).

- Patient-assessed intensity of symptoms score decreased significantly after six months from the baseline. It decreased by $32.3 \%$ for cramps; $32.4 \%$ for heaviness; $28.2 \%$ for pain; and $21.5 \%$ for swelling (Fig. 5).

- In patients with pedal oedema, leg circumference decreased after six months of treatment with MPFF (Fig. 6).

- MPFF appears to be extremely safe. Quality of life in the physical, social, and psychological domains was improved by $50 \%$, which is important because the majority of patients were in an active occupation.

- Six months of MPFF at $1000 \mathrm{mg}$ daily treatment without compression stockings or surgery is effective and acceptable in reversing the progression, ameliorating symptoms, and improving the QoL of patients with long standing CVIL.

- MPFF may be a useful agent in the overall management of CVI, particularly in countries with a tropical climate where clinical experience suggests that long-term compression treatment is difficult to comply with [10].

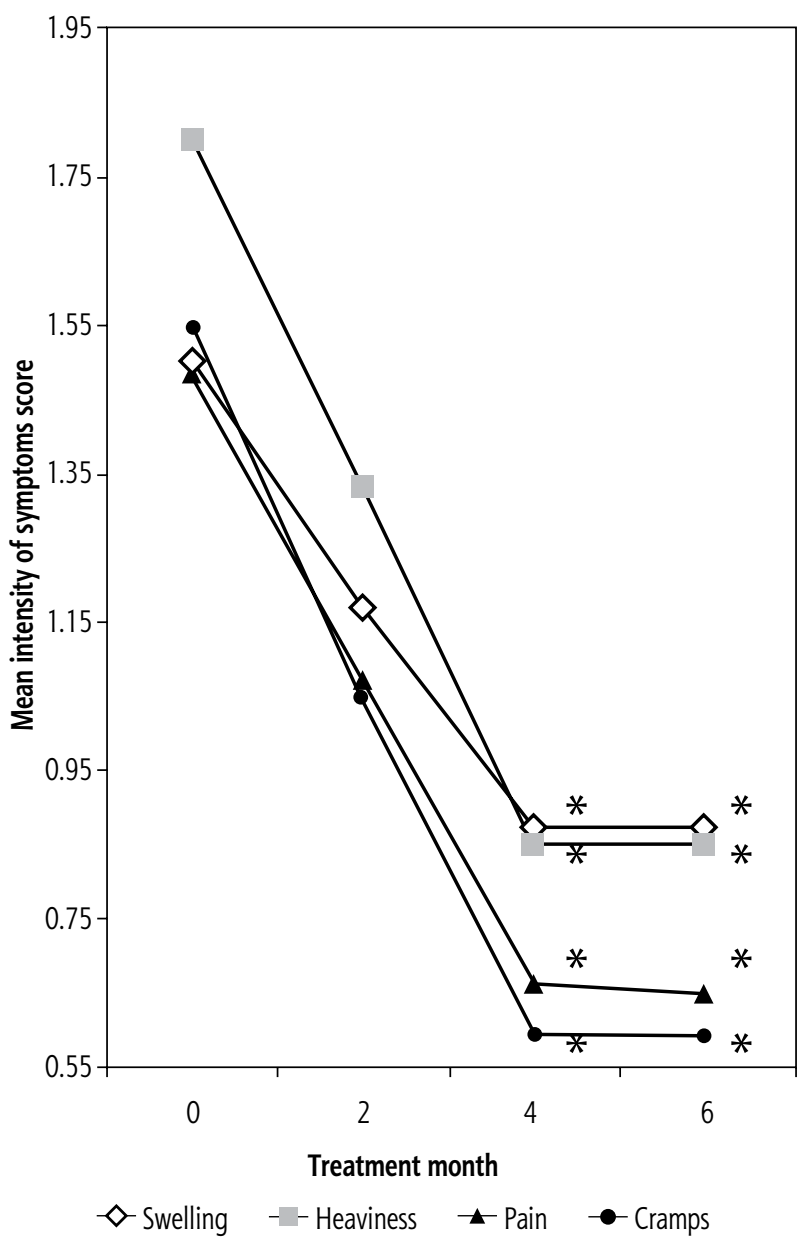

Fig. 5. Mean decrease in patient-assessed intensity of symptoms with MPFF 


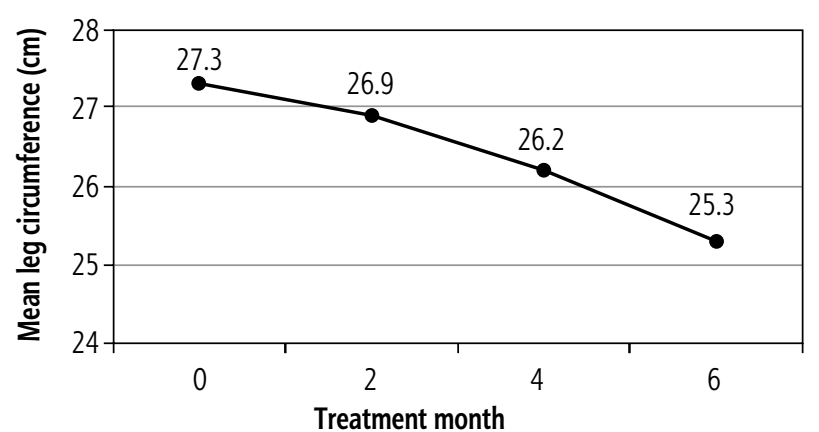

Fig. 6. Mean decrease in leg circumference in chronic venous insufficiency of the leg patients with oedema, after six months of treatment with MPFF

\section{Use of micronised purified flavonoid fraction}

- A meta-analysis of five RCTs evaluating ulcer healing in adults receiving one tablet of MPFF $1000 \mathrm{mg}$ a day in addition to standard treatment using a random-effects model showed a statistically significant effect in favour of MPFF [24].

- Another meta-analysis assessed the effect of oral treatment with MPFF on leg ulcer healing. It was observed that the chance for ulcer healing in the MPFF group compared to the controls was higher (Fig. 7) [25].

- The relative hazard of healing was $38 \%$ higher in the MPFF group compared to the control group. The curve of the cumulative percentage of patients who had healed their ulcer over time (Fig. 8) indicates a significantly shorter time to healing in the MPFF group compared with the control group. Thus, the results showed that venous ulcer healing is accelerated by MPFF treatment and MPFF might be a useful adjunct to conventional therapy in large and long standing ulcers [25].

\section{Horse chestnut seed extract (HCSE)}

- The horse chestnut tree (Aesculus hippocastanum) belongs to the same family as the sweet chestnut and Ohio buckeye trees.

- The most active ingredients of the extract are called escins (a- and b-escin).

- Studies have shown that HCSE inhibits serum lysosomal enzymes, which are important in the pathogenesis of CVI. By inhibiting these enzymes, HCSE may decrease capillary permeability and fragility [26].

- Ten studies compared HCSE against placebo, two against compression therapy, and five against other VADs. The studies reported that HCSE was significantly superior to placebo (Table 2) [27].

\section{Calcium dobesilate}

- Calcium dobesilate (2,5-dihydroxybenzene sulphonate) is a synthetic veno-active drug used in the treatment of

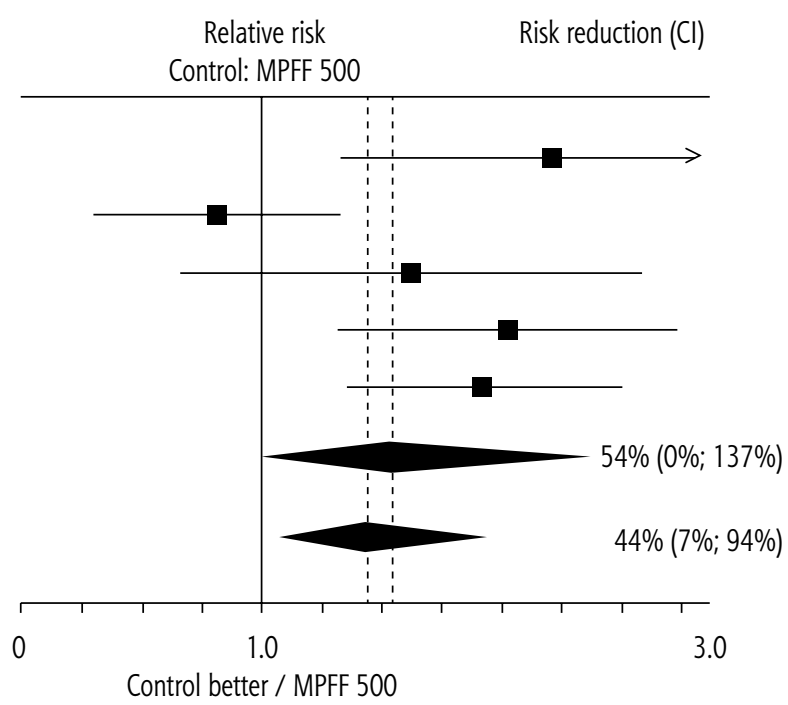

Fig. 7. Reduction of the relative risk of ulcer healing at 2 months in trials comparing MPFF with control

chronic venous insufficiency; it also helps in reducing cramps and restless legs. Calcium dobesilate decreases capillary permeability, as well as platelet aggregation and blood viscosity. Recently published data showed that calcium dobesilate increases endothelium-dependent relaxation owing to an increase in nitric oxide synthesis [28].

- Rabe et al. in a double-blind, placebo-controlled trial evaluated the efficacy of calcium dobesilate $(\mathrm{CaD})$ in chronic venous insufficiency (CVI) and concluded that $\mathrm{CaD}$ reduces pedal oedema and improves the symptoms of CVD independently of the concomitant usage of compression stockings [29].

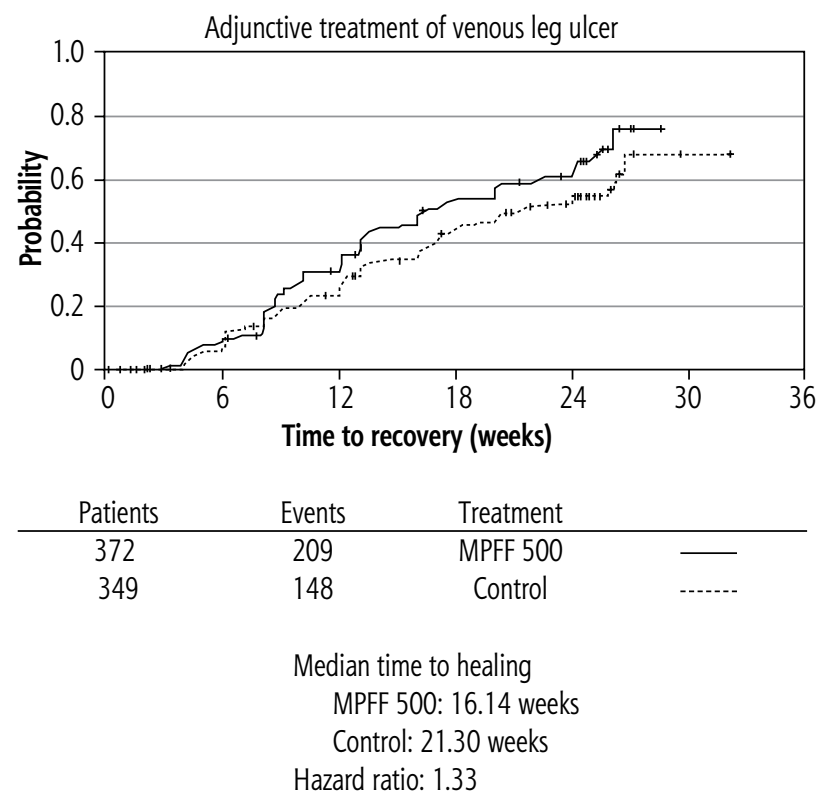

Fig. 8. Comparison of cumulative healing rates between the micronised purified flavonoid fraction group (solid line) and the control group (broken line). 
Table 2. Cochrane review of horse chestnut seed extract for CVI

\begin{tabular}{lccc}
\hline Outcome variable & N studies & N patients & Effect size \\
\hline Leg pain, responder ratio (D) & 1 & 418 & OR 2.22 [1.50; 3.29] \\
\hline Leg pain, VAS (C) & 1 & 30 & WMD 42.4 [34.9; 49.9] \\
\hline Oedema, responder ratio & 1 & 346 & OR 2.78 [1.79; 4.30] \\
\hline Oedema, VAS (C) & 1 & 30 & WMD 40.1 [31.6; 48.6] \\
\hline Lower leg volume (C) & 6 & 502 & WMD 32.1 [13.5; 50.7] \\
\hline Ankle circumference (C) & 3 & 80 & WMD 4.71 [1.13; 8.28] \\
\hline Calf circumference (C) & 3 & 80 & WMD 3.51 [0.58; 6.45] \\
\hline Itching, responder ratio (D) & 1 & 196 & OR 1.98 [1.11; 3.53] \\
\hline
\end{tabular}

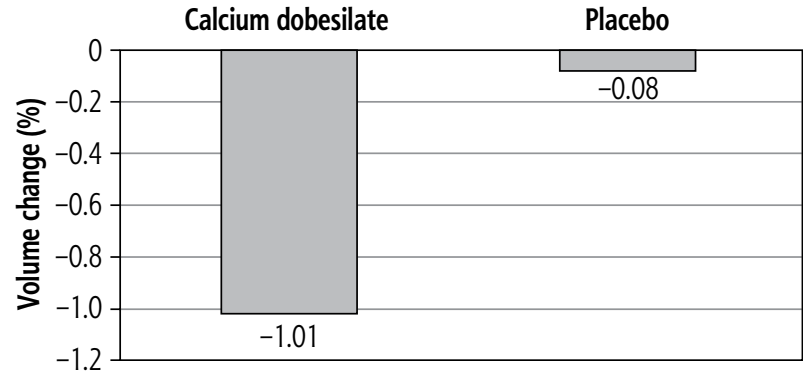

Fig. 9. Volume decrease in the most pathological leg with calcium dobesilate vs. placebo

- In another recent study published in 2015, Rabe et al. evaluated the superiority of $500 \mathrm{mg}$ calcium dobesilate given three times daily for 12 weeks versus placebo in reduction of oedema of the lower limbs in patients with CVI. At the end of 12-week follow-up, calcium dobesilate group had shown a significantly greater volume decrease in the most pathological leg (Fig. 9) [30].

However, calcium dobesilate has been associated with possible increased risk of agranulocytosis, a rare but serious condition [27]. The dose of 500-1000 mg/day of calcium dobesilate has been associated with side effects such as gastrointestinal disturbances, drug fever, and agranulocytosis [31] (Table 3).

\section{Compression therapy}

Compression therapy still remains the cornerstone of conservative treatment. Therapeutic leg compression includes elastic stockings and elastic bandages (short and long stretch). The elastic stockings and bandages act on superficial and deep veins and improve the muscle pump function, leading to reduction of ambulatory venous pressure and reduction of oedema [18].

\section{Chronic venous disease without ulceration (CO-C4)}

- Elastic stockings (in the form of graduated compression) have been used for the conservative management of CVD C0-C4 for decades. They compress varicose veins, reduce venous reflux, and improve calf muscle pump function.

- Recent studies have shown that progressive graduated compressive stockings (higher pressure at the calf compared with the ankle) are more effective than the usual digressive graduated compressive stockings (higher pressure at the ankle) in improving pain and lower leg symptoms.

- It was also observed that progressive graduated compressive stockings have a more pronounced effect on venous pumping function than graduated elastic compression.

Table 3. Summary of veno-active drugs

\begin{tabular}{lccc}
\hline Name of veno-active drug & Dosage & Advantages & Side effects \\
\hline MPFF & $1000 \mathrm{mg}$ daily & $\begin{array}{c}\text { Improves venous tone and lymphatic } \\
\text { drainage, and reduces capillary hyper } \\
\text { permeability }\end{array}$ & Gastrointestinal disturbances \\
\hline Pentoxifylline & $400 \mathrm{mg}$ TID & $\begin{array}{c}\text { Inhibits platelet aggregation; reduces blo- } \\
\text { od viscosity and improves microcirculation }\end{array}$ & $\begin{array}{c}\text { Nausea, vomiting, diarrhoea, heart- } \\
\text { burn, loss of appetite }\end{array}$ \\
\hline HCSE & $\begin{array}{l}\text { Do mg escins PO BID, with a maximum } \\
\text { daily dose of } 150 \mathrm{mg} / \text { day }\end{array}$ & $\begin{array}{c}\text { Act as anti-exudative and anti-inflammato- } \\
\text { ry agents; decreases vascular permeability } \\
\text { and thus prevents oedema formation }\end{array}$ & $\begin{array}{c}\text { Pruritus, nausea, dyspepsia, headache, } \\
\text { and dizziness }\end{array}$ \\
\hline Calcium dobesilate & $500-1000 \mathrm{mg} /$ day & $\begin{array}{c}\text { Decreases capillary permeability, as well as } \\
\text { platelet aggregation and blood viscosity }\end{array}$ & $\begin{array}{c}\text { Gastrointestinal disturbances, drug } \\
\text { fever, and agranulocytosis }\end{array}$ \\
\hline
\end{tabular}


- Elastic stockings require proper fitting and should be replaced at intervals of 3-4 months, to remain effective [18] (Table 4).

\section{Venous ulceration (C5-C6)}

\section{Venous ulcer healing}

- It was observed that compression bandages improve the healing rate of ulcers, compared with standard care without compression.

- Non-elastic compression is an alternative to traditional bandaging, which includes the Unna boot (a compression dressing impregnated with zinc oxide paste) and a non-elastic compression system based on adjustable Velcro bands that can be changed and adjusted daily eliminating odorous secretions.

- However, involving an elastic component in a bandage system seems more effective in terms of wound healing than when it is not involved.

- Sustained compression of at least $40 \mathrm{mmHg}$ with a four-layer compression bandage over a week has been shown to be more effective than lower grades of compression [18].

\section{Venous ulcer recurrence}

- Compression is important to prevent ulcer recurrence, and most benefit is seen from high compression; however, this is more likely to be associated with patient intolerance.

- Following healing of the ulcer, compression bandages should be replaced by elastic stockings.

- The latter could be the definitive treatment, particularly in patients with deep vein occlusion or gross incompetence not amenable to widely accepted surgical or interventional solutions.

- Patients should wear the highest level of compression that is comfortable, preferably $25-35 \mathrm{mmHg}$ at ankle level.

- In the ESCHAR trial, compression in the form of four-layer bandaging was shown to be as effective as surgery and compression in achieving healing of venous ulcers (Table 5).

\section{Intermittent pneumatic compression for venous ulceration}

- Intermittent pneumatic compression (IPC) improves venous flow significantly in patients using elastic bandages, while, in combination with elastic stockings, it has been shown to increase healing rates and overall healing of venous ulcers compared with elastic stockings alone.

- IPC is also effective in CVI, and higher compression pressures have been reported to be associated with greater leg volume reduction in patients with chronic venous oedema [18] (Table 6).

\section{Contraindications in the recommendation for use of compression therapy in the treatment of venous leg ulcers}

Studies investigating the efficacy of compression therapy were carried out in people without diabetes, cardiovascular disease, malignancy, or mixed aetiology ulcers. Compression should be used with greater caution in this population and may be contraindicated in high risk patients.

High-risk patients may include patients with:

- Heart failure;

- Peripheral arterial disease;

- Ankle brachial pressure index $(<0.8 \mathrm{mmHg}$ or $>1.2$ mmHg);

- Peripheral neuropathy;

- Some vasculitic ulcers.

Even though compression therapy may relieve lower limb oedema, the aetiology should be determined and the patient's condition monitored closely. Consider compression therapy only in patients who can detect increasing pain or complica-

Table 4. Grades of recommendations for CVD without ulceration [18]

\begin{tabular}{lc}
\hline Recommendations & Grades of recommendations \\
\hline $\begin{array}{l}\text { Elastic stockings are recommended as an } \\
\text { effective treatment modality for symp- } \\
\text { toms and signs of chronic venous disease }\end{array}$ \\
\hline Temporary use of elastic stockings may be \\
considered in patients with chronic venous \\
disease awaiting further investigation, and \\
as a definite treatment in patients who are \\
not managed by invasive methods \\
\hline
\end{tabular}

Table 5. Grades of recommendations for venous ulceration [18]

\begin{tabular}{|c|c|}
\hline Recommendations & Grades of recommendations \\
\hline $\begin{array}{l}\text { Compression bandages and walking } \\
\text { exercises are recommended as the initial } \\
\text { treatment modality to promote healing in } \\
\text { patients with venous leg ulcers }\end{array}$ & A \\
\hline $\begin{array}{l}\text { The use of high compression pressure of at } \\
\text { least } 40 \mathrm{mmHg} \text { at the ankle level should } \\
\text { be considered, to promote ulcer healing }\end{array}$ & B \\
\hline
\end{tabular}

Table 6. Grades of recommendations for IPC [18]

\begin{tabular}{lc}
\hline Recommendations & Grades of recommendations \\
\hline IPC is recommended to provide sympto- \\
matic relief in patients with CVD (C3-C6), \\
if standard methods are not indicated or if \\
they have failed \\
\hline IPC should be considered in patients with \\
venous ulcers after a 6-month treatment \\
course when standard methods have failed
\end{tabular}


tions and for whom the compression system can promptly be removed, e.g. by the patient or another person [32].

Potential modifications in the high-risk patient include:

- Increased frequency of review by a health professional specialised in venous leg ulcer management;

- Increased frequency of assessment for signs and symptoms of complications, e.g. tissue necrosis, skin trauma, discolouration, pain, pallor, paraesthesia, impaired capillary return;

- Reduction in the level of compression;

- Increased padding/comfort layer under the compression [33].

\section{Surgical treatments for CVI}

\section{Sclerotherapy}

- Injection sclerotherapy can be used for superficial varicose veins, residual or recurring varicose veins, following surgery and thread veins to obliterate the varicose vein.

- Initially sodium tetradecyl sulphate (STD) is injected into the affected blood vessel and pressure pad dressings are applied at the injection site, after which compression bandages are applied.

- Complications of sclerotherapy include formation of blood clots, skin staining, inflammation, ulcers, tissue damage, and reactions to the sclerosing agent [34].

\section{Endovenous treatments}

- Endovenous techniques in the treatment of saphenous vein incompetence have become very popular as a minimally invasive alternative to classical surgery.

- In the last decade, endovenous techniques have evolved quickly and performance has improved. Endovenous treatment options include endovenous thermal ablation (EVTA), mechano-chemical ablation (MOCA), and injection of cyanoacrylate glue [18].

\section{Endovenous thermal ablation (EVTA)}

- The two most frequently used EVTA techniques are endovenous laser ablation (EVLA) and radiofrequency ablation (RFA). Other less common techniques include steam ablation.

- EVLA and RFA have the same occlusion rates, but patients treated with RFA have less postoperative pain and bruising.

- Compared with surgery, EVTA seems to be more efficient and results in fewer post-operative side effects (less paraesthesia and pain, and a faster return to normal activities) [18].

\section{Mechano-chemical endovenous ablation}

- In this technique, endomechanical abrasion is produced by the tip of the catheter's rotating wire (mechanical component) and endovenous chemical ablation via the simultaneous injection of sclerosant over the rotating wire (chemical component).

- This technique is under development because the optimal dosage of sclerosants still needs to be determined [18].

\section{PERSPECTIVE ON PREVENTION OF CHRONIC VENOUS DISEASE PROGRESSION}

\section{Lifestyle adaptation}

For mild forms of venous disease, lifestyle changes may be recommended to control existing symptoms and prevent others.

The following measures may help prevent varicose veins and CVI:

- Managing blood pressure and body weight;

- Regular exercise like running or walking;

- Leg elevation;

- Avoiding prolonged standing or sitting;

- Avoiding clothes that are tight around the waist, thighs, or legs;

- Strengthening of calf muscles and avoiding shoes that limit the use of calf muscles, for example high heels [35].

\section{Pharmacological}

- A large number of naturally occurring and synthetic agents have been shown to have veno-active properties, and in many countries the use of veno-active drugs (VADs) is generally considered as an adjunct to sclerotherapy or surgery.

- MPFF is one of the widely used treatments to relieve symptoms of chronic venous insufficiency. Of particular interest is the action of MPFF at a dose of 1000 mg on inflammatory reactions associated with venous hypertension, inhibition of leukocyte activation, adhesion, and migration.

- Horse chestnut seed extract (HCSE) has a similar mode of action as MPFF but has fewer clinical benefits than MPFF.

- Pentoxifylline plus compression therapy was found to be more effective than placebo plus compression for ulcer healing. The current use of this drug is, however, limited by the frequent occurrence of side effects [36].

\section{Compression}

- Compression bandages are long strips of fabric that are wrapped around the leg to form a continuous covering. - These bandages are applied so that the long edges overlap to prevent gaps. Compression therapy systems may be categorised according to the pressure produced on a model limb at the ankle during laboratory testing.

- An international consensus group has recommended a system for categorising compression bandage systems (Table 7). 
- Compression hosiery (stocking) that delivers at least $18-24 \mathrm{mmHg}$ and up to $35 \mathrm{mmHg}$ at the ankle has been recommended for the prevention of venous leg ulcers [37] (Table 8).

\section{Surgery}

- Surgical management of CVI may be considered to complement the compressive stocking in those refractory to medical and endovenous therapy.

- Surgery for truncal veins is directed toward preventing the consequences of reflux in the superficial venous system by interruption and removal.

- In a study evaluating 500 patients with venous ulcer and reflux of superficial and deep venous systems, random assignment to surgery (only to the superficial venous segments) plus compression demonstrated a significant reduction in ulcer recurrence at 12 months compared with compression alone (12\% vs. 28\%) [39] (Table 9).

\section{INDIAN EXPERTS' PROPOSAL OF ANOTHER WAY OF UNDERSTANDING THE DISEASE}

- In spite of various methods of reporting/classifying the disease, it has become inadequate to understand the disease pattern.

- The Indian experts propose another way of understanding the disease as congenital and acquired CVD.

- Acquired could be either primary acquired CVD or secondary (post thrombotic syndrome) acquired CVD.

- This consensus intentionally focuses only on the acquired CVD. One of the biggest risk factors for CVD is prolonged standing. Certain occupations that require people to stand for a long time (like nurses, teachers, traffic police, office-workers, housewives, etc.) increase the risk of acquired CVD.

- In the female gender there is an increased risk of post-partum CVD.

- Therefore, this makes Part I of the consensus statement. Based on this we will conduct a nationwide study of the disease prevalence, pattern, and epidemiological survey to give a guideline useful for the Indian set up and many countries other than India having diversified conditions.

\section{CONCLUSIONS}

In India, awareness of the warning symptoms of CVD among the general population is far from satisfactory. This lack of awareness leads to a delay in receiving medical advice from local doctors and a delay in hospitalisation, which are setbacks for providing the necessary treatment. A high prevalence of CVD has been documented in India and hence studies need to be conducted in different parts of India to develop a National Chronic Venous registry that would define criteria, dietary, and risk fac-
Table 7. Recommended categorisation of compression bandage systems

\begin{tabular}{cc}
\hline Category & Pressure $(\mathrm{mmHg})$ \\
\hline Mild & $<20$ \\
\hline Moderate & $\geqslant 20-40$ \\
\hline Strong & $\geqslant 40-60$ \\
\hline Very strong & $\geqslant 60$ \\
\hline
\end{tabular}

Table 8. Grades of recommendations for preventing ulcer recurrence [38]

\begin{tabular}{lc}
\hline \multicolumn{1}{c}{ Recommendations } & Grades of recommendations \\
\hline Below-knee graduated compression hosie- \\
ry is recommended to prevent recurrence \\
of venous leg ulcer in patients where leg \\
ulcer healing has been achieved
\end{tabular}

Table 9. Grades of recommendations for surgical management [38]

\begin{tabular}{lc}
\hline Recommendations & Grades of recommendations \\
\hline Patients with chronic venous leg ulcer and & B \\
superficial venous reflux should be con- \\
sidered for superficial venous surgery to \\
prevent recurrence
\end{tabular}

tors because India is a multi-ethnic and multicultural country. There is now an urgent need undertake health education measures about the awareness of risk factors and early warning signs of CVD in the community so that people in general and affected subjects specifically can receive early and effective therapy through modern means. A holistic approach to contain the challenge of CVD in India is absolutely essential because the illness is now poised to become a major problem in this country.

Primary care physicians (PCPs) who see patients as part of their practice play a central role in advancing the understanding of venous disease. When patients understand their disease and are informed about options for treatment, they are more likely to have a positive interaction with their provider, receive quality care, and improve their quality of life. For more patients to receive an accurate diagnosis and effective treatment for venous disease, an increase in the clinicians' education is required. The educational process should be directed towards clinicians in primary care clinics, nurse practitioner, physician assistant-run centres, and even the emergency room.

The role of the primary care physician in this process is critical because treatment at an early stage can prevent complications like venous ulcers. A major educational program is needed to enrol primary care clinicians as key members of the team to reduce venous complications through recognition of these early cases. The educational message to pri- 
mary providers is that chronic venous disease is a lifelong progressive degenerative process, much like peripheral arterial disease. Effective management prior to irreversible skin changes will prevent marked disability in the patient's later life. A strong public awareness campaign is also needed to alert patients with progressive venous disease to contact their physician for diagnostic evaluation and definitive care. Too often, the patient's early inquiry may be overlooked by the general physician as a minor problem, which can delay definitive evaluation and preventive management.

\section{The authors declare no conflict of interest.}

\section{References}

1. Pitsch F. The place of Micronized Purified Flavonoid Fraction in recent international guidelines on the management of symptomatic chronic venous disorders. Medicographia 2015; 37: 71-79.

2. Mukunda N.K. Clinical evaluation and management of lower limb varicose veins: A study at KIMS. Unpublished doctoral dissertation submitted to Rajiv Gandhi University of Health Sciences; 2006

3. Eklof B., Perrin M., Delis K.T., Rutherford R.B., Gloviczki P. Updated terminology of chronic venous disorders: The VEINTERM transatlantic interdisciplinary consensus document. J Vasc Surg 2009; 49: 498-501.

4. Halim I., Omar A.R. A review on health effects associated with prolonged standing in the industrial workplaces. IJRRAS 2011; 8: 14-21.

5. Prajapati P., Modi K., Rahul K., Shah A. A study related to effects of job experience on health of traffic police personnel of Ahmedabad City, Gujarat, India. IJIMS 2015; 2: 127-133.

6. Franks P.J., Wright D., McCollum C.N. Epidemiology of venous disease: a review. Phlebology 1989; 4: 143-151.

7. Jantet G. Chronic venous insufficiency: worldwide results of the RELIEF study. Angiology 2002; 53: 245-256.

8. Agale S.V. Chronic leg ulcers: epidemiology, aetiopathogenesis, and management. Ulcers 2013; Article ID: 413604.

9. Malhotra S.L. An epidemiological study of varicose veins in Indian railroad workers from the south and north of India, with special reference to the causation and prevention of varicose veins. Int J Epidemiol 1972; 1: 177-183.

10. Pinjala RK, Abraham TK, Chadha SK, Hai A.A, Hussain S.A., Moulik A.K., Nagori L.F., Nayak G., Patel M.D., Sen G., Shetty S.V., Sinha K.N. Long-term treatment of chronic venous insufficiency of the leg with micronized purified flavonoid fraction in the primary care setting of India. Phlebology 2004; 19: 179-184.

11. Launois R., Reboul-Marty J., Henry B. Construction and validation of a quality of life questionnaire in chronic lower limb venous insufficiency (CIVIQ). Qual Life Res 1996; 5: 539-554.

12. Milic D.J. Prevalence and socioeconomic data in chronic venous disease: how useful are they in planning appropriate management? In: Chronic venous disease guidelines and daily clinical practice. 2011; 33: 253-258.

13. Ramamoorthy P. The Growing Threat of Chronic Venous Disease [Internet]. Available from: http://www.apiindia.org/medicine_update_2013/chap173 (Cited on February 3, 2016).

14. Venous Insufficiency and Varicosities: Nutritional Considerations [Internet]. Available from:http://www.nutritionmd.org/ health_care_providers/cardiovascular/varicose_nutrition.html (Cited on February 3, 2016).

15. Padberg F. Jr, Cerveira J.J., Lal B.K., Pappas P.J., Varma S., Hobson R.W. 2nd. Does severe venous insufficiency have a different etiology in the morbidly obese? Is it venous? J Vasc Surg 2003; 37: 79-85.

16. What causes varicose veins: top 5 jobs that require prolonged standing or sitting? [Internet]. Available from: http://www. nutritionmd.org/health_care_providers/cardiovascular/varicose_nutrition.html (Cited on February 3, 2016).

17. Eklöf B., Rutherford R.B., Bergan J.J., Carpentier P.H., Gloviczki P., Kistner R.L., Meissner M.H., Moneta G.L., Myers K., Padberg F.T., Perrin M., Ruckley C.V., Smith P.C., Wakefield T.W. Revision of the CEAP classification for chronic venous disorders: Consensus statement. J Vasc Surg 2004; 40: 1248-1252.

18. Wittens C., Davies A.H., Bækgaard N., Broholm R., Cavezzi A., Chastanet S., de Wolf M., Eggen C., Giannoukas A., Gohel M., Kakkos S., Lawson J., Noppeney T., Onida S., Pittaluga P., Thomis S., Toonder I., Vuylsteke M., Kolh P., de Borst G.J., Chakfé N., Debus S., Hinchliffe R., Koncar I., Lindholt J., de Ceniga M.V., Vermassen F., Verzini F., De Maeseneer M.G., Blomgren L., Hartung O., Kalodiki E., Korten E., Lugli M., Naylor R., Nicolini P., Rosales A. Editor's Choice: Management of Chronic Venous Disease Clinical Practice Guidelines of the European Society for Vascular Surgery (ESVS). Eur J Vasc Endovasc Surg 2015; 49: 678-737.

19. Reeder S.W., Roos K.P., Neumann H.A.M. Chronic venous disease under pressure. September $25^{\text {th }}, 2013$.

20. Krishnan S., Nicholls S.C. Chronic venous insufficiency: clinical assessment and patient selection. Semin Intervent Radiol 2005; 22: 169-177.

21. Eberhardt R.T., Raffetto J.D. Chronic venous insufficiency. Circulation 2005; 111: 2398-2409.

22. Lyseng-Williamson K.A., Perry C.M. Micronised purified flavonoid fraction: a review of its use in chronic venous insufficiency, venous ulcers and haemorrhoids. Drugs 2003; 63: 71-100.

23. Gohel M.S., Davies A.H. Pharmacological agents in the treatment of venous disease: an update of the available evidence. Curr Vasc Pharmacol 2009; 7: 303-308.

24. Scallon C., Bell-Syer S.E., Aziz Z. Flavonoids for treating venous leg ulcers. Cochrane Database Syst Rev 2013; 5: CD006477.

25. Coleridge-Smith P., Lok C., Ramelet A.A. Venous leg ulcer: a meta-analysis of adjunctive therapy with micronized purified flavonoid fraction. Eur J Vasc Endovasc Surg 2005; 30: 198-208.

26. Szapary P.O., Cirigliano M.D. Horse chestnut seed extract for the treatment of chronic venous insufficiency. AHC Media 1999; 2: 25-28.

27. Bergan J., Paquette N.B. The vein book. $2^{\text {nd }}$ ed. Oxford University Press, Oxford 2014.

28. Tejerina T., Ruiz E. Calcium dobesilate: pharmacology and future approaches. Gen Pharmacol 1998; 31: 357-360.

29. Rabe E., Jaeger K.A., Bulitta M., Pannier F. Calcium dobesilate in patients suffering from chronic venous insufficiency: a double-blind, placebo-controlled, clinical trial. Phlebology 2011; 26: 162-168.

30. Rabe E., Ballarini S., Lehr L.; Doxium EDX09/01 Study Group. A randomized, double-blind, placebo-controlled, clinical study on the efficacy and safety of calcium dobesilate in the treatment of chronic venous insufficiency. Phlebology 2016; 31: 264-274.

31. Duggal L., Thukral R., Kumar S. Calcium D6obesilate-Induced Agranulocytosis. Available from: http://www.japi.org/ april2005/Correspondence.pdf (Cited on February 3, 2016). 
32. Smith P.D. Micronized purified flavonoid fraction and the treatment of chronic venous insufficiency: microcirculatory mechanisms. Microcirculation 2000; 7 (6 Pt 2): S35-40.

33. Australian and New Zealand Clinical Practice Guideline for Prevention and Management of Venous Leg Ulcers. Available from: httphttp://www.awma.com.au/publications/2011_awma_ vlu_guideline_abridged.pdf (Cited on February 3, 2016).

34. Tisi P.V., Beverley C., Rees A. Injection sclerotherapy for varicose veins. Cochrane Database Syst Rev 2006; (4): CD001732.

35. Lifestyle Changes for Chronic Venous Insufficiency. Available from: http://nyulangone.org/conditions/chronic-venous-insufficiency/treatments/lifestyle-changes-for-chronic-venous-insufficiency (Cited on February 3, 2016).

36. Pharmacological treatment in patients with chronic venous disease. ActaDermatovenerol Croat 2012; 20: 197-214.

37. Principles of compression in venous disease: A practitioner's guide to treatment and prevention of venous leg ulcers. Available from: http://www.woundsinternational.com/media/ issues/672/files/content_10802.pdf (Cited on February 3, 2016).

38. Management of chronic venous leg ulcers a national clinical guideline. Available from: http://www.sign.ac.uk/pdf/sign120. pdf (Cited on February 3, 2016).

39. Eberhardt R.T., Raffetto J.D. Chronic venous insufficiency. Circulation 2014; 130: 333-346. 